\title{
Can strangelets solve the muon puzzle?
}

\author{
Maciej Rybczyński ${ }^{1, *}$ and Zbigniew Włodarczyk ${ }^{1, * *}$ \\ ${ }^{1}$ Institute of Physics, Jan Kochanowski University, Kielce, Poland
}

\begin{abstract}
In order to examine a muon excess observed by the Pierre Auger Observatory, detailed Monte Carlo simulations were carried out, assuming the existence of strangelets (hypothetical stable lumps of strange quark matter) in the primary cosmic rays. We obtain a rough agreement between the simulations and the data for ordinary nuclei without any contribution of strangelets in the primary flux of cosmic rays.
\end{abstract}

\section{Introduction}

Over the past few decades, it has been suspected that the number of registered muons at the surface of the Earth is tens of percentage points higher than what it should be, according to existing hadronic interaction models $[1,2]$. Recently, a study from the Pierre Auger Collaboration (Auger) has strengthened this suspicion, using a novel technique to mitigate some of the measurement uncertainties of earlier methods [1,3]. The new analysis of Auger data suggests that the hadronic component of showers with primary energy $E>10^{18} \mathrm{eV}$ contains about $30 \%$ to $60 \%$ more muons than expected. Also the number of muons with energies above $0.75 \mathrm{GeV}$, determined by the Sydney University Giant Air-shower Recorder (SUGAR) [4], exceeds the simulated one by the factors $\sim 1.67$ and $\sim 1.28$ for $10^{17} \mathrm{eV}$ proton and iron primaries, respectively. The muon excess grows moderately with the primary energy, increasing by an additional factor of 1.2 for $10^{18.5} \mathrm{eV}$ primaries. On the other hand, very recently, no muon excess in EAS has been reported. The comparison of observed and simulated densities of $E_{\mu}>10 \mathrm{GeV}$ muons in air showers induced by $E \sim 10^{17} \mathrm{eV}$ primaries, based on the Moscow State University Extensive Air Shower (EASMSU) data [5], demonstrate that the muon excess is absent and that the fit never requires primaries heavier than iron. To explain the muon excess, several new models have been proposed, exploring new physics [6-8] or new forms of matter, namely strange quark matter (SQM) [9]. In this paper we adopt a purely phenomenological approach to develop an SQM scheme. In sharp contrast to previous models our approach is based on the assumption that ultrahigh energy cosmic rays are very heavy strange quark objects, i.e. strangelets.

\section{Strangelets in cosmic rays}

The analysis of the EAS data offers a unique possibility to observe possible imprints of strangelets arriving from

\footnotetext{
*e-mail: Maciej.Rybczynski@ujk.edu.pl

**e-mail: Zbigniew.Wlodarczyk@ujk.edu.pl
}

outer space. They are lumps of SQM, a new possible stable form of matter (cf. [10-12] for details). Following [13] it is fully reasonable to search for SQM in cosmic ray experiments because the specific features of strangelets $[14,15]$ allow them to penetrate deep into the atmosphere $[16,17]$. The point is that there is a certain critical size of the strangelet, given by the critical value of its mass number $A=A_{\text {crit }} \simeq 300-400$, such that for $A>A_{\text {crit }}$ strangelets are absolutely stable against neutron emission. Below this limit strangelets decay rapidly evaporating neutrons. The spatial radii of strangelets turn out to be comparable to the radii of ordinary nuclei [13], i.e., their geometrical cross sections are similar to the normal nuclear ones. To account for their strong penetrability one has to accept that strangelets penetrating deeply into the atmosphere are formed in many successive interactions with the air nuclei by the initially very heavy lumps of SQM entering the atmosphere and decreasing due to the collisions with air nuclei (until their $A$ reaches the critical value $A_{\text {crit }}$ [13]). Such scenario is fully consistent with all present experiments [13, 16-18]. In this scenario the interaction of a strangelet of mass $A$ with an air nucleus of mass $A_{\text {air }}$ involves all quarks of the target located in the geometrical intersection of the colliding air nucleus and the strangelet. So, up to $3 \cdot A_{\text {air }}$ quarks from the strangelet could be used making its mass drop to a value of $A-A_{\text {air }}$.

There are several reports suggesting the existence of direct candidates for SQM [19, 20] (characterized mainly by their very small $Z / A$ ratios). All of them have mass numbers A near or slightly exceeding $A_{\text {crit }}$. Analysis of these candidates for SQM show $[13,16,17]$ that the abundance of strangelets in the primary cosmic ray flux is $F_{S Q M}\left(A=A_{\text {crit }}\right) / F_{\text {tot }} \simeq 2.4 \cdot 10^{-5}$ at the same energy per particle. The expected flux of strangelets is then equal to $F_{S Q M} \simeq 7 \cdot 10^{-6} \mathrm{~m}^{-2} \mathrm{~h}^{-1} \mathrm{sr}^{-1}$ for the energy above $10 \mathrm{GeV}$ per initial strangelet, which is consistent with the astrophysical limits and with the upper limits given by the experiment [21]. The data follows the $A^{-7.5}$ behaviour, which coincides with the behaviour of abundance of normal nuclei in the Universe [22]. 


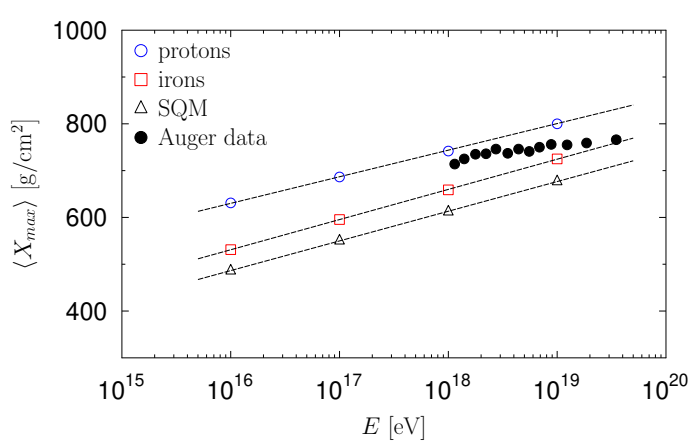

Figure 1. Average position of the shower maximum as a function of the primary energy for EAS generated by protons, iron nuclei, and SQM. Dotted lines show a fit of the type $\left\langle X_{\max }\right\rangle=c+d$. $\ln \left(E / 10^{19} \mathrm{eV}\right)$. Pierre Auger Collaboration measurements [29] are indicated by black circles.

Experimental results show a wide spectrum of exotic events (Centauros, superfamilies with 'halo', strongly penetrating component, etc.) which are clearly incompatible with the standard ideas of hadronic interactions known from accelerator experiments. Some new mechanism or new primaries are therefore needed. Assuming that strangelets represent such new primaries one is able to explain the long-flying component of cosmic rays. Also muon bundles of extremely high multiplicity, observed recently by the ALICE detector at LHC, in its dedicated cosmic-ray run [23] (confirming similar finding from the LEP era at CERN [24]) detectors cosmic-ray run, can originate from collisions of strangelets with the atmosphere [17, 25]. All these considerations motivate further experimental search for the SQM and for its cosmological and elementary particle physics aspects.

\section{EAS simulations}

For the simulation of the propagation of extensive air showers in the Earth's atmosphere we have used a suitably modified SHOWERSIM [26] modular software. In our simulations we used the F00 model which consists in a simple non-scaling extrapolation of the inclusive data at ISR and SPS energies [27]. In this version, the leading particle remembers its charge, and the $x$-distribution of secondaries does not depend on the elasticity (relative energy carried by the leading particle). For collisions with air nuclei, the inelastic cross-section dependence on energy is given by: $\sigma_{\text {inel }}=\sigma_{0}+\delta \cdot\left[\ln \left(E / E_{0}\right)\right]^{\alpha}$ for $E \geq E_{0}$ and $\sigma_{\text {inel }}=\sigma_{0}$ for $E<E_{0}$, where $\sigma_{0}=280 \mathrm{mb}, \delta=2.5$ $\mathrm{mb}$ and $E_{0}=0.1 \mathrm{TeV}$ for primary nucleons. In the F00 model the inelasticity $K$, i.e. the relative energy used for production of secondaries, is sampled from a uniform distribution over an $(0,1)$ interval for interacting nucleons and over an $(0.333,1)$ interval for meson interactions. Therefore, the average inelasticity $\langle K\rangle=0.5$ for nucleons and $\langle K\rangle=0.667$ for mesons. In the development of EAS, the inelasticity $K$ and the cross section for interactions $\sigma$ are strongly correlated. The attenuation of hadrons or the depth of the shower maximum $X_{\max }$ are actually the measure of combinations of $K$ and $\sigma$, and the effect of these

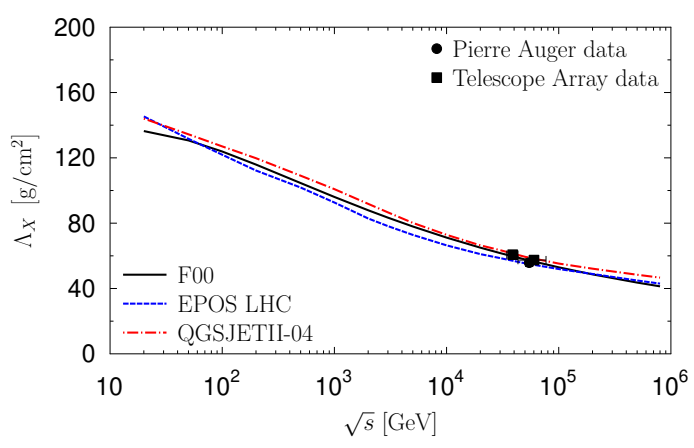

Figure 2. Attenuation length as a function of the center-ofmass energy for our F00 model (full line). EPOS LHC (dotted line) and QGSJETII-04 (dash-dotted line) predictions are taken from [28]. Pierre Auger Collaboration measurements [30] are indicated by black circles while Telescope Array data [31] by black squares.

two parameters is extremely difficult to disentangle. From the above we can see that $\langle K\rangle$ and $\sigma_{\text {inel }}$ differ significantly for different models. Nevertheless, the $\left\langle X_{\max }\right\rangle$ for these models are quite similar.

The average positions of showers maxima as a function of primary energy are shown in figure 1. Obviously, the simulated values for different primaries follow a logarithmic trend, thus were fitted using the formula $\left\langle X_{\max }\right\rangle=$ $c+d \cdot \ln \left(E / 10^{19} \mathrm{eV}\right)$, with $c=801.4,724.3,683.8$ and $d=24.7,28.0,27.7$ for protons, iron nuclei and SQM, respectively. The deep tail of the depth of maximum distribution, which has an exponential behaviour: $d N / d X_{\max } \sim$ $\exp \left(-X_{\max } / \Lambda_{X}\right)$ depends on the proton interaction length $\lambda=14.45 m_{p} / \sigma_{\text {inel }}=2.4 \cdot 10^{4} / \sigma_{\text {inel }}\left[\mathrm{g} / \mathrm{cm}^{2}\right]$ via shower maxima attenuation length: $\Lambda_{X} \simeq 0.8 \lambda / K$. Figure 2 shows the shower maxima attenuation length as a function of the center-of-mass energy for our F00 model (full line). EPOS LHC (dotted line) and QGSJETII-04 (dash-dotted line) predictions are taken from reference [28]. Despite the very different energy dependence of $\langle K\rangle$ and $\sigma_{\text {inel }}$, the shower maxima attenuation lengths $\Lambda_{X}$ are very similar. We evaluated the attenuation of the showers maxima, $\Lambda_{X}$, for protons at $10^{18} \mathrm{eV}$ obtaining $\Lambda_{X}=58.5 \pm 1 \mathrm{~g} / \mathrm{cm}^{2}$, which agrees nicely with Pierre Auger Collaboration [30] and Telescope Array [31] experimental data, see figure 2.

We performed Monte Carlo simulations of the EAS generated by primary nuclei (protons and iron nuclei) and by primary strangelets with mass $A \geq A_{\text {crit }}=320$ taken from the $A^{-7.5}$ distribution. In this study we have generated the inclined events (primaries were sampled with zenith angle $\Theta$ from the interval $62^{\circ}-80^{\circ}$ and abreviation average $\Theta=67^{\circ}$ was used for simplicity) with energies in the interval $10^{16}<E<10^{19} \mathrm{eV}$. In the analysis we have focused on the muons in the nuclear cascade with energies larger than $0.3 \mathrm{GeV}$, which is the Cherenkov threshold for muons in water, reaching the Pierre Auger South Laboratory surface detector placed at the altitude $1425 \mathrm{~m}$ above sea level. This corresponds to a total atmospheric depth of $X_{\text {atm }}=750 \mathrm{~g} / \mathrm{cm}^{2}$ for the showers initiated at $\Theta=0^{\circ}$. 


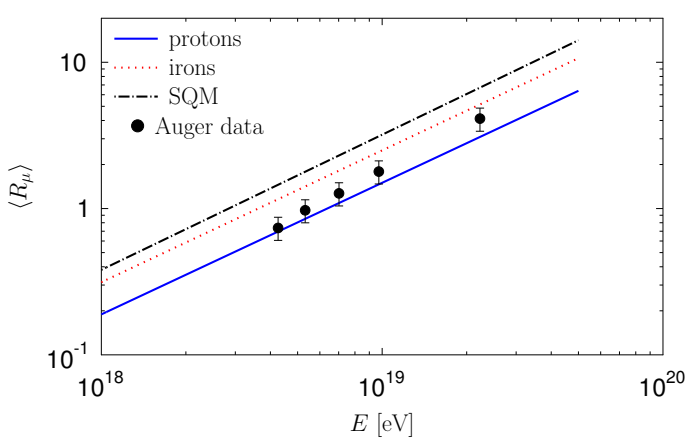

Figure 3. The average muon contents $\left\langle R_{\mu}\right\rangle$ of individual showers generated by primary protons, iron nuclei, and SQM, plotted as a function of the primary energy $E$, together with the Pierre Auger Collaboration results taken from [1]. See text for details

\section{Results}

In this section we provide a comparison of our SHOWERSIM simulations with the Pierre Auger Collaboration results presented in reference [1]. We focus at the showers generated with zenith angles $\Theta=670$. The Pierre Auger Collaboration presents the measured number of muons in inclined air showers using the scaled factor, relating the observed muon densities at the ground to the average muon density of simulated EAS induced by protons at a fixed energy $10^{19} \mathrm{eV}$. The used scale factor is independent from the zenith angle and from the details of the location of the detector [32, 33]. Following [1] the muon content $R_{\mu}$ is defined as:

$$
R_{\mu}=N_{\mu} / N_{\mu, 19}
$$

where $N_{\mu}$ is the total number of muons at the ground in EAS generated by primary cosmic rays at different primary energies, and $N_{\mu, 19}$ is the total number of muons in EAS generated by primaries with energy $E=10^{19} \mathrm{eV}$. Using equation 4 and following [1] we used the power-law parametrization:

$$
\left\langle R_{\mu}\right\rangle=a \cdot\left(E / 10^{19} \mathrm{eV}\right)^{b}
$$

with parameters $a=1.841$ and $b=1.029$ fitting Auger experimental events above $4 \cdot 10^{18} \mathrm{eV}$. At zenith angle $\Theta=67^{\circ}$ the muon content $R_{\mu}=1$ corresponds to $N_{\mu}=1.455 \cdot 10^{7}$ muons at the ground with energies above $0.3 \mathrm{GeV}$. For model comparisons, as described in [1], the simulated number of muons should be then divided by $N_{\mu}=1.455 \cdot 10^{7}$ to obtain $R_{\mu}$, which can be directly compared to Auger measurements. In figure 3 we show the simulated muon content $\left\langle R_{\mu}\right\rangle$ of individual showers generated by primary protons, iron nuclei, and SQM, as a function of the primary energy $E$. In figure 3 we show the power-law fits to our simulated events (prepared using equation 2 with the parameters: $a=1.5,2.5,3.2$ and $b=$ $0.9,0.9,0.92$ for protons, iron nuclei, and SQM resprctively. Note that the parameters obtained in our SHOWERSIM simulations are very close to those obtained from Auger experiment, see reference [1]. We also show in figure 3 the corresponding Auger measurement [1]. It is noticeable that Auger results are located between the simu-

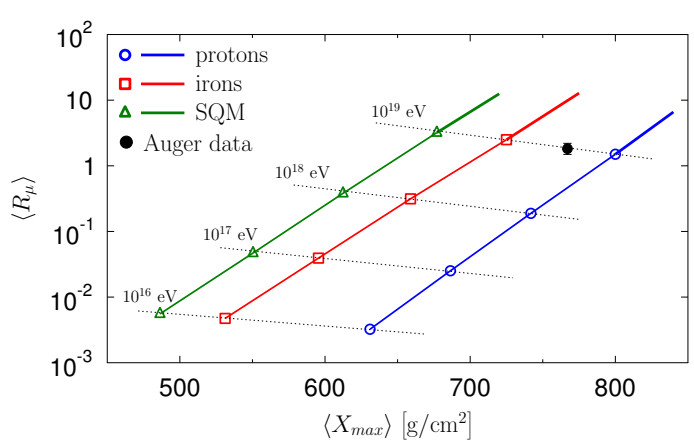

Figure 4. Average muon content $\left\langle R_{\mu}\right\rangle$ of individual showers generated by primary protons, iron nuclei, and SQM as a function of the average position of the shower maximum. The full circle shows the Auger result [1]. Dotted lines connect simulations performed at the same primary energy.

lation of protons and iron nuclei. At lower energies, the experimental points are close to the $\left\langle R_{\mu}\right\rangle$ values obtained in the simulations assuming protons as primaries. However, at higher energies, the measured muon content approaches the $\left\langle R_{\mu}\right\rangle$ values coming from simulations of EAS generated by iron nuclei. The dependence of the average muon content $\left\langle R_{\mu}\right\rangle$ on the position of the showers maximum is presented in figure 4 . It is remarkable that no heavier component than iron is needed to describe the experimental values of $\left\langle R_{\mu}\right\rangle$ at $10^{19} \mathrm{eV}$. We can explain experimental data without strange quark matter.

The muon content in the air shower, $R_{\mu}$, is a quantity related to the atomic mass $A$ of the primary cosmic ray. A possible implication for the mass composition is demonstrated on the example, taking into account the obtained experimentally $\left\langle R_{\mu}\right\rangle=1.82 \pm 0.38$ and the relative standard deviation $\omega=\sigma\left(R_{\mu}\right) /\left\langle R_{\mu}\right\rangle=0.20 \pm 0.01$ [1]. For a single component, the values of $\omega$ can vary between $\omega=0.04$ for pure iron nuclei and $\omega=0.13$ for protons. More than two components (proton and iron) are needed to describe the first two moments of the $R_{\mu}$ distribution. The best description of the data is obtained with four components (40\% protons, $20 \%$ helium, 35\% nitrogen, and 5\% iron nuclei) while the addition of more species does not improve the quality of the fit. The comparatively small abundance of iron nuclei has been evaluated from the analysis of the $\left\langle X_{\max }\right\rangle-\sigma\left(X_{\max }\right)$ difference [34, 35]. Our results suggest roughly $\langle\ln A\rangle=1.4$ with standard deviation $\sigma(\ln A)=1.3$ at energies above $4 \cdot 10^{18} \mathrm{eV}$.

\section{Concluding remarks}

The mass composition of cosmic rays can be derived from certain air shower observables, but the inference is limited by our theoretical understanding of the air shower development. Air shower simulations require knowledge of hadronic interaction properties at very high energies and in phase space regions that are not well covered by accelerator experiments. The systematic uncertainty of the inferred mass composition can be reduced by studying different observables. The slant depth of the shower maximum $X_{\max }$ 
is a prominent mass-sensitive tracer, since it can be measured directly with fluorescence telescopes. The average depth of shower maximum is

$$
\left\langle X_{\max }\right\rangle=X_{0} \ln \left[\left(E_{0} K\right) /(N \epsilon)\right]-X_{0} \ln A+\left\langle X_{1}\right\rangle,
$$

where $X_{0}=37 \mathrm{~g} / \mathrm{cm}^{2}$ is the radiation length in air, $\epsilon=81$ $\mathrm{MeV}$ is the critical energy in air, $\left\langle X_{1}\right\rangle$ is the mean depth of the interaction with maximal energy deposition within the shower (usually called the depth of the first interaction), $K$ denotes inelasticity and $\langle N\rangle$ is related to the multiplicity of secondaries in the high-energy hadronic interactions in the cascade. The number of muons in an air shower is another powerful tracer of the mass. Simulations show that the number of produced muons, $N_{\mu}$, rises almost linearly with the cosmic-ray energy, $E$, and increases with a small power of the cosmic-ray mass, $A$. This behaviour can be understood in terms of the generalized Heitler model of hadronic air showers [36], which predicts

$$
N_{\mu}=A^{1-\beta}\left(E / \xi_{c}\right)^{\beta},
$$

where $\xi_{c}$ is the critical energy at which charged pions decay into muons, and $\beta \approx 0.9$. The dependence of the muon number, $N_{\mu}$, on the mass of the primary cosmic rays is complementary to the depth of the shower maximum, $X_{\max }$. If both observables are combined, the internal consistency of the mass composition can be tested.

Very surprisingly the F00 model included in the SHOWERSIM simulation package [26] describe nicely the muon content in EAS. Ordinary nuclei, without any contribution from strange quark matter in the primary flux of cosmic rays, can describe experimental data. Even if the strangelets contribute with a small amount in the primary flux and generate high multiplicity muon bundles, as we advocated recently [17], their influence on the average muon content $\left\langle R_{\mu}\right\rangle$ in EAS is negligible.

\section{Acknowledgements}

MR was supported by the Polish National Science Centre (NCN) grant 2016/23/B/ST2/00692.

\section{References}

[1] A. Aab et al. [Pierre Auger Collaboration], Phys. Rev. D 91, no. 3, 032003 (2015)

[2] T. Abu-Zayyad et al. [HiRes and MIA Collaborations], Phys. Rev. Lett. 84, 4276 (2000)

[3] A. Aab et al. [Pierre Auger Collaboration], Phys. Rev. Lett. 117, no. 19, 192001 (2016)

[4] J. A. Bellido et al., Phys. Rev. D 98, 023014 (2018)

[5] Y. A. Fomin et al., Astropart. Phys. 92, 1 (2017)

[6] G. R. Farrar and J. D. Allen, EPJ Web Conf. 53, 07007 (2013)
[7] J. Allen and G. Farrar, arXiv:1307.7131 [astro-ph.HE]

[8] J. Alvarez-Muniz, L. Cazon, R. Conceicao, J. D. de Deus, C. Pajares and M. Pimenta, arXiv:1209.6474 [hep-ph]

[9] L. A. Anchordoqui, H. Goldberg and T. J. Weiler, Phys. Rev. D 95, no. 6, 063005 (2017)

[10] R. Klingenberg, J. Phys. G 25, R273 (1999)

[11] E. Witten, Phys. Rev. D 30, 272 (1984)

[12] C. Alcock and A. Olinto, Ann. Rev. Nucl. Part. Sci. 38, 161 (1988)

[13] G. Wilk and Z. Wlodarczyk, J. Phys. G 22, L105 (1996)

[14] C. Alcock and E. Farhi, Phys. Rev. D 32, 1273 (1985)

[15] E. Farhi and R. L. Jaffe, Phys. Rev. D 30, 2379 (1984)

[16] G. Wilk and Z. Wlodarczyk, Nucl. Phys. Proc. Suppl. 52B, 215 (1997)

[17] P. Kankiewicz, M. Rybczynski, Z. Włodarczyk and G. Wilk, Astrophys. J. 839, no. 1, 31 (2017)

[18] G. Wilk and Z. Wlodarczyk, Acta Phys. Hung. A 4, 395 (1996)

[19] T. Saito, Y. Hatano, Y. Fukada and H. Oda, Phys. Rev. Lett. 65, 2094 (1990)

[20] P. B. Price, E. K. Shirk, R. Hagstrom and W. Z. Osborne, Phys. Rev. D 18, 1382 (1978)

[21] Z.Sahnoun et al. [SLIM Collaboration], Radiation Measurements 44, 894 (2009)

[22] G. B. Zhdanov, Usp. Fiz. Nauk, 111, 109 (1973)

[23] J. Adam et al. [ALICE Collaboration], JCAP 1601, no. 01, 032 (2016)

[24] V. Avati et al., Astropart. Phys. 19, 513 (2003)

[25] M. Rybczynski, Z. Wlodarczyk and G. Wilk, Nucl. Phys. Proc. Suppl. 97, 85 (2001)

[26] A. Wrotniak, SHOWERSIM/84 (University of Maryland Preprint, 1984)

[27] J. A. Wrotniak and G. B. Yodh, in Proc. 19th International Cosmic Ray Conference, 1985656

[28] T. Pierog, EPJ Web Conf. 145, 18002 (2017)

[29] J. Abraham et al. [Pierre Auger Collaboration], Phys. Rev. Lett. 104, 091101 (2010)

[30] P. Abreu et al. [Pierre Auger Collaboration], Phys. Rev. Lett. 109, 062002 (2012)

[31] R. Abbasi, EPJ Web Conf. 120, 04005 (2016)

[32] M. Ave, R. A. Vazquez, E. Zas, J. A. Hinton and A. A. Watson, Astropart. Phys. 14, 109 (2000)

[33] A. Aab et al. [Pierre Auger Collaboration], arXiv:1307.5059 [astro-ph.HE]

[34] G. Wilk and Z. Wlodarczyk, J. Phys. G 38, 085201 (2011)

[35] G. Wilk and Z. Włodarczyk, in Proc. 32th International Cosmic Ray Conference, 2011227

[36] J. Matthews, Astropart. Phys. 22, 387 (2005) 